\title{
ON SUMS OF POWERS OF INVERSE COMPLETE QUOTIENTS
}

\author{
OLIVER JENKINSON
}

(Communicated by Jane M. Hawkins)

\begin{abstract}
For an irrational number $x$, let $x_{n}$ denote its $n$-th continued fraction inverse complete quotient, obtained by deleting the first $n$ partial quotients. For any positive real number $r$, we establish the optimal linear bound on the sum of the $r$-th powers of the first $n$ complete quotients. That is, we find the smallest constants $\alpha(r), \beta(r)$ such that $x_{1}^{r}+\ldots+x_{n}^{r}<\alpha(r) n+\beta(r)$ for all $n \geq 1$ and all irrationals $x$.
\end{abstract}

\section{INTRODUCTION}

Every irrational number $x$ has a unique expansion as an infinite continued fraction

$$
x=\left[a_{0} ; a_{1}, a_{2}, a_{3}, \ldots\right]=a_{0}+\frac{1}{a_{1}+\frac{1}{a_{2}+\frac{1}{a_{3}+\cdots}}}
$$

where each partial quotient $a_{i}$ is an integer, and $a_{i} \geq 1$ for $i \geq 1$. Let

$$
x_{n}=\left[0 ; a_{n}, a_{n+1}, a_{n+2}, \ldots\right]=\frac{1}{a_{n}+\frac{1}{a_{n+1}+\frac{1}{a_{n+2}+\cdots}}}
$$

denote the $n$-th inverse complete quotient ${ }^{1}$ of $x$, obtained by deleting the first $n$ partial quotients in its continued fraction expansion. Each $x_{n}$ is an irrational number in the interval $(0,1)$.

For any positive real number $r$, we shall be concerned with the growth of the sum

$$
x_{1}^{r}+\ldots+x_{n}^{r}
$$

as $n \rightarrow \infty$. The growth is at most linear, and the purpose of this note is to identify the optimal constants $\alpha(r)$ and $\beta(r)$ for which ${ }^{2}$

$$
x_{1}^{r}+\ldots+x_{n}^{r}<\alpha(r) n+\beta(r)
$$

Received by the editors January 3, 2007.

2000 Mathematics Subject Classification. Primary 26Dxx; Secondary 11A55, 37D20, 37E05.

${ }^{1}$ See e.g. $[2, \S 10.9, \quad$ p. 139$]$ for the definition of the $n$-th complete quotient as $\left[a_{n} ; a_{n+1}, a_{n+2}, \ldots\right]$, the reciprocal of $x_{n}=\left[0 ; a_{n}, a_{n+1}, a_{n+2}, \ldots\right]$.

${ }^{2}$ It turns out that $\alpha(r) n+\beta(r)$ is a strict upper bound on $x_{1}^{r}+\ldots+x_{n}^{r}$, though this is not a priori obvious. 
for all $n \geq 1$ and all irrationals $x$.

More precisely, we define $\alpha(r)$ and $\beta(r)$ by

$$
\alpha(r)=\sup _{x \in \mathbb{R} \backslash \mathbb{Q}} \limsup _{n \rightarrow \infty}\left(x_{1}^{r}+\ldots+x_{n}^{r}\right) / n
$$

$\operatorname{and}^{3}$

$$
\beta(r)=\sup _{x \in \mathbb{R} \backslash \mathbb{Q}} \sup _{n \in \mathbb{N}}\left(x_{1}^{r}+\ldots+x_{n}^{r}\right)-\alpha(r) n
$$

and prove:

Theorem 1. For all irrationals $x$ and all $n \geq 1$,

$$
x_{1}^{r}+\ldots+x_{n}^{r}< \begin{cases}(n-1) \gamma^{r}+1 & \text { if } 0<r \leq r^{*}, \\ (n+1) / 2 & \text { if } r \geq r^{*},\end{cases}
$$

where $\gamma=(\sqrt{5}-1) / 2 \approx 0 \cdot 618$ and $r^{*}=-\log 2 / \log \gamma \approx 1 \cdot 4404$.

These inequalities are sharp, in the sense that $\beta(r)=1-\alpha(r)$ for all $r>0$, and

$$
\alpha(r)= \begin{cases}\gamma^{r} & \text { for } 0<r \leq r^{*} \\ 1 / 2 & \text { for } r \geq r^{*}\end{cases}
$$

Remark 1. The novelty in Theorem 1 is that the bound on $x_{1}^{r}+\ldots+x_{n}^{r}$ holds for all irrationals, and is sharp. This should be compared with, on the one hand, trivial sub-optimal bounds such as $x_{1}^{r}+\ldots+x_{n}^{r}<n$, and on the other hand with the equality

$$
\lim _{n \rightarrow \infty} \frac{1}{n}\left(x_{1}^{r}+\ldots+x_{n}^{r}\right)=\frac{1}{\log 2} \int_{0}^{1} \frac{t^{r}}{1+t} d t=\frac{1}{\log 2} \sum_{k=1}^{\infty} \frac{(-1)^{k+1}}{r+k}
$$

which is valid for Lebesgue almost every irrational. This latter result follows from the pointwise ergodic theorem applied to the (ergodic) dynamical system $([0,1], T, G)$, where $T:[0,1] \rightarrow[0,1]$ is defined (Lebesgue almost everywhere) by $T(x)=1 / x(\bmod 1)$, and $G$ denotes Gauss measure $d G(t)=(\log 2)^{-1} d t /(1+t)$ (see e.g. [1, p. 174]). For example if $r=1$, then the righthand side of (2) equals $(\log 2)^{-1}-1 \approx 0 \cdot 44$, which is strictly less than $\alpha(1)=\gamma=(\sqrt{5}-1) / 2 \approx 0 \cdot 618$.

The key to Theorem 1 is the following result, which will be proved in $\S 2$.

Theorem 2. Let $\left(x_{i}\right)_{i=1}^{\infty}$ be a sequence of reals in $[0,1]$ such that $x_{i}\left(1+x_{i+1}\right) \leq 1$ for all $i \geq 1$. If $0<r \leq r^{*}$, then $x_{1}^{r}+\ldots+x_{n}^{r} \leq(n-1) \gamma^{r}+1$ for all $n \geq 1$.

\section{Proof of Theorems 1 And 2} by

In order to prove Theorem 2 , suppose that $0<r \leq r^{*}$, and define $\varphi_{r}:[0,1] \rightarrow \mathbb{R}$

$$
\varphi_{r}(x)= \begin{cases}1-2 \gamma^{r} & \text { if } 0 \leq x \leq c_{r} \\ -x^{r} & \text { if } c_{r} \leq x \leq \gamma \\ -\gamma^{r} & \text { if } \gamma \leq x \leq 1\end{cases}
$$

where we set

$$
c_{r}:=\left(2 \gamma^{r}-1\right)^{1 / r} \geq 0
$$

\footnotetext{
${ }^{3}$ It is not immediately obvious that $\beta(r)$ is finite, though this turns out to be the case.
} 
Note in particular that $\varphi_{r}$ is non-increasing, is strictly decreasing on $\left[c_{r}, \gamma\right]$, and that its global oscillation is

$$
\operatorname{Osc}\left(\varphi_{r}\right):=\sup _{x, y \in[0,1]}\left|\varphi_{r}(x)-\varphi_{r}(y)\right|=1-\gamma^{r} .
$$

We first claim that if $x, y \in[0,1]$ satisfy $x(1+y) \leq 1$, then

$$
x^{r}-\gamma^{r} \leq \varphi_{r}(y)-\varphi_{r}(x) .
$$

If $0 \leq x \leq \gamma$, then (3) clearly holds, because $\varphi_{r}(x) \leq-x^{r}$ and $\varphi_{r}(y) \geq \min \varphi_{r}=$ $-\gamma^{r}$.

To establish (3) when $\gamma \leq x \leq 1$, first note that

$$
\varphi_{r}(x)=-\gamma^{r},
$$

and $y \leq x^{-1}-1 \leq \gamma^{-1}-1=\gamma$. Since $\varphi_{r}$ is non-increasing, and $x^{-1}-1 \in[0, \gamma]$,

$$
\varphi_{r}(y) \geq \varphi_{r}\left(x^{-1}-1\right)=\min \left(1-2 \gamma^{r},-\left(x^{-1}-1\right)^{r}\right),
$$

and combining it with (4) gives

$$
\varphi_{r}(y)-\varphi_{r}(x)-x^{r}+\gamma^{r} \geq \min \left(1-x^{r}, 2 \gamma^{r}-x^{r}-\left(x^{-1}-1\right)^{r}\right) .
$$

To prove (3) we must show that the righthand side of $(5)$ is non-negative. Since $1-x^{r} \geq 0$, this is the case if and only if $\left(x^{-1}-1\right)^{r}+x^{r} \leq 2 \gamma^{r}$, and writing $z=x^{-1}-1$ this is equivalent to proving that

$$
z^{r}+(1+z)^{-r}-2 \gamma^{r} \leq 0 \quad \text { for all } z \in[0, \gamma], r \in\left(0, r^{*}\right] .
$$

Define $F_{r}(z):=z^{r}+(1+z)^{-r}-2 \gamma^{r}$. Since $F_{r}(\gamma)=0$, the inequality (6) follows if $F_{r}$ is increasing on $[0, \gamma]$, and for $0<r \leq 1$ this is the case: its derivative $r z^{r-1}-r(1+z)^{-(r+1)}$ is positive because $z^{r-1} \geq 1$ and $(1+z)^{r+1}>1$. It remains to prove (6) when $1<r \leq r^{*}$ : in this case

$$
r^{-1} F_{r}^{\prime \prime}(z)=(r-1) z^{r-2}+(r+1)(1+z)^{-(r+2)}>0,
$$

so $F_{r}$ is convex on $[0, \gamma]$, and therefore $(6)$ follows from the fact that $F_{r}(0)=$ $1-2 \gamma^{r} \leq 0$ and $F_{r}(\gamma)=0$. $(3)$,

Now suppose the sequence $\left(x_{i}\right)_{i=1}^{\infty}$ satisfies $x_{i}\left(1+x_{i+1}\right) \leq 1$ for each $i \geq 1$. From

(7) $x_{1}^{r}+\ldots+x_{n}^{r} \leq n \gamma^{r}+\varphi_{r}\left(x_{n+1}\right)-\varphi_{r}\left(x_{1}\right) \leq n \gamma^{r}+\operatorname{Osc}\left(\varphi_{r}\right)=(n-1) \gamma^{r}+1$

for all $n \geq 1$, and Theorem 2 is proved.

We wish to use Theorem 2 to deduce Theorem 1, first in the case $0<r \leq r^{*}$. Note that if $\left(x_{i}\right)_{i=1}^{\infty}$ is the sequence of inverse complete quotients of some irrational, then $x_{i} \in(0,1)$, and $x_{i+1}=x_{i}^{-1}-a_{i} \leq x_{i}^{-1}-1$, for each $i \geq 1$. So $\left(x_{i}\right)_{i=1}^{\infty}$ satisfies the hypotheses of Theorem 2, and therefore (7) holds for $0<r \leq r^{*}$. In particular, (7) implies that $\alpha(r) \leq \gamma^{r}$. Now the constant sequence $(\gamma, \gamma, \ldots)$, which is the sequence of inverse complete quotients for the number $\gamma$, attains the supremum in the definition of $\alpha(r)$, so in fact $\alpha(r)=\gamma^{r}$. From this, and (7), it follows that $\beta(r) \leq 1-\gamma^{r}$. But

$$
\beta(r)=\sup _{x \in \mathbb{R} \backslash \mathbb{Q}} \sup _{n \in \mathbb{N}}\left(x_{1}^{r}+\ldots+x_{n}^{r}\right)-\alpha(r) n \geq \sup _{x \in \mathbb{R} \backslash \mathbb{Q}} x_{1}^{r}-\alpha(r)=1-\gamma^{r},
$$

so in fact $\beta(r)=1-\gamma^{r}$. 
To show that (7) is always a strict inequality, note that equality first implies that $\varphi_{r}\left(x_{i+1}\right)=x_{i}^{r}-\gamma^{r}+\varphi_{r}\left(x_{i}\right)$ for all $1 \leq i \leq n$, in particular

$$
\varphi_{r}\left(x_{n+1}\right)=x_{n}^{r}-\gamma^{r}+\varphi_{r}\left(x_{n}\right),
$$

and second that $\varphi_{r}\left(x_{n+1}\right)-\varphi_{r}\left(x_{1}\right)=\operatorname{Osc}\left(\varphi_{r}\right)=1-\gamma^{r}$, hence

$$
x_{n+1} \in\left[0, c_{r}\right] .
$$

But now if $x_{n} \in\left[0, c_{r}\right)$, then (8) means $\varphi_{r}\left(x_{n+1}\right)<c_{r}^{r}+1-3 \gamma^{r}=-\gamma^{r}=$ $\min \varphi_{r}$, a contradiction, while if $x_{n} \in\left[c_{r}, \gamma\right]$, then (8) becomes $\varphi_{r}\left(x_{n+1}\right)=-\gamma^{r}$, hence $x_{n+1} \in[\gamma, 1]$, contradicting (9), and finally if $x_{n} \in(\gamma, 1)$, then (8) means that $\varphi_{r}\left(x_{n+1}\right)=x_{n}^{r}-2 \gamma^{r}<1-2 \gamma^{r}$, which implies that $x_{n+1} \in\left(c_{r}, 1\right]$, again contradicting (9). So we have shown that $x_{n}$ does not lie in $[0,1)$, a contradiction; therefore $(7)$ is a strict inequality when $\left(x_{i}\right)_{i=1}^{\infty}$ is the sequence of inverse complete quotients of an irrational.

Turning now to the case $r>r^{*}$, note that $r \mapsto x^{r}$ is strictly decreasing for every $x \in(0,1)$, so $r \mapsto \lim \sup _{n \rightarrow \infty}\left(x_{1}^{r}+\ldots+x_{n}^{r}\right) / n$ is non-increasing for every $x \in \mathbb{R} \backslash \mathbb{Q}$, and hence

$$
\alpha(r)=\sup _{x \in \mathbb{R} \backslash \mathbb{Q}} \limsup _{n \rightarrow \infty} \frac{x_{1}^{r}+\ldots+x_{n}^{r}}{n}
$$

is non-increasing in $r$. In particular,

$$
\alpha(r) \leq \alpha\left(r^{*}\right)=\gamma^{r^{*}}=1 / 2 \text { for all } r>r^{*} .
$$

Now let $x=[0 ; 1,1,1,2,1,3, \ldots]$ be the irrational whose partial quotients $a_{i}$ are given by $a_{i}=1$ for $i$ odd, and $a_{i}=i / 2$ for $i$ even. Then

$$
x_{2 i-1}=T^{2 i-2}(x)=[0 ; 1, i, \ldots] \rightarrow 1 \quad \text { as } i \rightarrow \infty,
$$

whereas

$$
x_{2 i}=T^{2 i-1}(x)=[0 ; i, \ldots] \rightarrow 0 \quad \text { as } i \rightarrow \infty,
$$

so that

$$
\frac{x_{1}^{r}+\ldots+x_{n}^{r}}{n} \rightarrow \frac{1}{2} \quad \text { as } n \rightarrow \infty
$$

Therefore

$$
\alpha(r)=\sup _{x \in \mathbb{R} \backslash \mathbb{Q}} \limsup _{n \rightarrow \infty} \frac{x_{1}^{r}+\ldots+x_{n}^{r}}{n} \geq \frac{1}{2},
$$

and together with (10) we deduce that

$$
\alpha(r)=1 / 2 \text { for all } r>r^{*} .
$$

To show that $\beta(r)=1 / 2$, first note that $\beta(r)=\sup _{x \in \mathbb{R} \backslash \mathbb{Q}} \sup _{n \in \mathbb{N}}\left(x_{1}^{r}+\ldots+x_{n}^{r}\right)$ $-\alpha(r) n \geq \sup _{x \in \mathbb{R} \backslash \mathbb{Q}} x_{1}^{r}-\alpha(r)=1-\alpha(r)=1 / 2$. Now $\alpha\left(r^{*}\right)=1 / 2=\beta\left(r^{*}\right)$, and if $x$ is irrational, then $x_{1}^{r^{*}}+\ldots+x_{n}^{r^{*}}-\frac{n}{2}<\frac{1}{2}$ for $n \geq 1$, so if $r>r^{*}$, then

$$
x_{1}^{r}+\ldots+x_{n}^{r}-\frac{n}{2}<x_{1}^{r^{*}}+\ldots+x_{n}^{r^{*}}-\frac{n}{2}<\frac{1}{2}
$$

for $n \geq 1$, thereby establishing the second inequality in (1). Moreover we deduce that

$$
\beta(r)=\sup _{x \in \mathbb{R} \backslash \mathbb{Q}} \sup _{n \in \mathbb{N}}\left(x_{1}^{r}+\ldots+x_{n}^{r}\right)-\alpha(r) n \leq 1 / 2,
$$

and hence that $\beta(r)=1 / 2$ for all $r>r^{*}$, thus concluding the proof of Theorem 1 . 


\section{ACKNOWLEDGMENTS}

I am grateful to Thierry Bousch for helpful comments which shortened the proof, and to Dan Mauldin and Mariusz Urbański for helpful conversations. This research was partially supported by an EPSRC Advanced Research Fellowship and research grant GR/S50991/01.

\section{REFERENCES}

[1] I. P. Cornfeld, S. V. Fomin \& Ya. G. Sinai, Ergodic theory, Springer-Verlag, 1982. MR832433 (87f:28019)

[2] G. H. Hardy \& E. M. Wright, An introduction to the theory of numbers (Fifth edition), Oxford University Press, 1979. MR568909 (81i:10002)

School of Mathematical Sciences, Queen Mary, University of London, Mile End RoAd, London E1 4NS, United Kingdom

E-mail address: omj@maths.qmul.ac.uk

$U R L$ : www.maths.qmul.ac.uk/ omj 